\title{
Transcatheter arterial chemoembolization with gemcitabine and oxaliplatin for the treatment of advanced biliary tract cancer
}

This article was published in the following Dove Press journal:

OncoTargets and Therapy

9 March 2015

Number of times this article has been viewed

\section{Qing Zhao* \\ Sheng Qian* \\ Liang Zhu \\ Xu-Dong Qu \\ Wei Zhang \\ Zhi-Ping Yan \\ Jie-Min Cheng \\ Qing-Xin Liu \\ Rong Liu \\ Jian-Hua Wang}

Department of Interventional Radiology, Zhongshan Hospital, Fudan University, Shanghai, People's Republic of China

*These authors contributed equally to this work

Correspondence: Rong Liu; Jian-Hua Wang

Department of Interventional Radiology, Zhongshan Hospital, Fudan University, 180 Fenglin Road, Xuhui District, Shanghai 200032, People's Republic of China

Tel/fax +86 21 64041990 extension 3088 Email fdzsIr@I63.com; docwjh@I26.com
Background: The aim of this study was to determine the therapeutic efficacy and safety of transarterial chemoembolization (TACE) with gemcitabine and oxaliplatin in patients with advanced biliary tract cancer (BTC).

Methods: We retrospectively analyzed the outcomes for 65 patients with advanced BTC treated by TACE with gemcitabine $1,000 \mathrm{mg} / \mathrm{m}^{2}$ and oxaliplatin $100 \mathrm{mg} / \mathrm{m}^{2}$. Follow-up laboratory tests and computed tomography or magnetic resonance imaging were performed routinely to evaluate the response of the tumor to treatment. All patients were assessed for adverse effects.

Results: Of the 65 patients, 19 (29.2\%) achieved a partial response, 36 (55.4\%) showed stable disease, and ten (15.4\%) showed progressive disease. The overall response rate was $29.2 \%$. At the end of this study, five patients were still alive. The median overall survival was 12.0 months (95\% confidence interval 8.5-15.5). There were no serious complications after TACE.

Conclusion: The disease control rate and overall survival in this retrospective study were consistent with those in previous reports. TACE with gemcitabine and oxaliplatin was well tolerated and highly effective in patients with advanced BTC.

Keywords: gemcitabine, oxaliplatin, transcatheter arterial chemoembolization, biliary tract cancer

\section{Introduction}

Biliary tract cancers (BTCs), including intrahepatic cholangiocarcinoma, extrahepatic cholangiocarcinoma, and gallbladder cancer, are relatively rare tumors. ${ }^{1}$ At present, surgical resection is the only curative treatment, but at least $75 \%$ of cases are unresectable because of the advanced stage of disease at diagnosis. ${ }^{2,3}$ The median survival of patients with cholangiocarcinoma and gallbladder cancer is 3-6 months and 6 months, respectively. ${ }^{4,5}$ Factors influencing survival include the specific tumor location, margin status, lymph node metastasis, vascular invasion, histology, albumin level, and bilirubin level. ${ }^{6,7}$

Patients with locally advanced BTC can be considered for palliative chemotherapy. Several reports have concluded that, compared with gemcitabine alone, cisplatin plus gemcitabine significantly improves overall survival and progression-free survival in patients with locally advanced BTC.$^{8-11}$

Transcatheter arterial chemoembolization (TACE) is characterized by delivery of chemotherapeutic agents directly into the arteries supplying the tumor. TACE results in a higher concentration of chemotherapeutic drugs in the tumor and a lower concentration in normal tissues. Therefore, TACE can improve therapeutic efficacy and minimize systemic toxicity. In recent years, studies of the efficacy of TACE in 
patients with unresectable BTC have reported promising results. $^{12-18}$

To our knowledge, few reports are available on the efficacy and toxicity of TACE with gemcitabine and oxaliplatin in advanced BTC. In this retrospective study, we determined the therapeutic efficacy and safety of TACE with gemcitabine and oxaliplatin in the treatment of patients with advanced BTC.

\section{Patients and methods}

\section{Patient selection}

This retrospective analysis involved 65 patients who received TACE for advanced BTC between July 2009 and December 2013 in the Department of Interventional Radiology, Zhongshan Hospital, Fudan University. The diagnosis of BTC was confirmed by histological examination or by radiological and clinical findings. The inclusion criteria were as follows: BTC deemed to be unresectable, according to the National Comprehensive Cancer Network Guidelines (version 1.2009); Eastern Cooperative Oncology Group performance status of two or lower; lesions that could be evaluated using computed tomography or magnetic resonance imaging; adequate hematological and renal function (white blood cell count $>4 \times 10^{9} / \mathrm{L}$; platelet count $>100 \times 10^{9} / \mathrm{L}$ ); and total bilirubin, serum aspartate transaminase, and serum alanine transaminase concentrations less than 1.5 times the upper limit of normal. Exclusion criteria included extensive portal vein thrombosis and prior systemic chemotherapy or radiation therapy. The study was approved by the ethics committee of Zhongshan Hospital, Fudan University.

\section{TACE protocol}

A 5-French arterial sheath was placed into the right femoral artery under local anesthesia. A celiac arteriogram was obtained using a 4-French or 5-French catheter to evaluate the anatomy, tumor burden, and vascularity. After placement of the microcatheter (Progreat ${ }^{\mathrm{TM}}$, Terumo Corporation, Tokyo, Japan) in the artery supplying the tumor, we administered $100 \mathrm{mg} / \mathrm{m}^{2}$ oxaliplatin (Jiangsu Hengrui Medicine Co Ltd, Lianyungang, People's Republic of China) and 1,000 mg/m² gemcitabine (Jiangsu Haosen Medicine Co Ltd, Lianyungang, People's Republic of China). Next, a mixture of iodized oil (Lipiodol $^{\circledR}$, Laboratoire Guerbet, Aulny-sous-bois, France), oxaliplatin, and the nonionic contrast medium Ultravist 300 (Bayer Schering Pharma AG, Leverkusen, Germany) was infused into the selected feeding artery via the microcatheter until arterial flow stasis was achieved. The dosage of iodized oil depended on tumor size and number, blood supply, and liver function. Next, gelatin sponge particles $(350-510 \mu \mathrm{m}$, Ailikang Medicine Co Ltd, Hangzhou, People's Republic of China) diluted with the same contrast medium were injected through a $1 \mathrm{~mL}$ syringe. The specific dilution of the gelatin sponge particles depended on parameters such as the diameter of the artery feeding the tumor.

All patients received supportive treatments, including antibiotic prophylaxis, liver protection, antacid agents, and antiemetic treatment, after the TACE procedure. Follow-up laboratory tests (for example, blood cell count and liver function tests) were routinely performed at one-month intervals. Follow-up imaging was performed in all patients 4-6 weeks after each TACE treatment. The TACE treatments were repeated if a new or residual tumor was detected on follow-up imaging. We aimed to determine the impact of TACE in terms of response rate and overall survival. Tumor response after each TACE treatment was evaluated according to the Response Evaluation Criteria in Solid Tumors. Overall survival was measured from the date of diagnosis to the date of death or the last follow-up. Adverse effects were evaluated according to the Common Terminology Criteria for Adverse Events version 3.0.

\section{Statistical analysis}

The Kaplan-Meier method was used to evaluate overall survival. The Student's $t$-test was used to compare continuous data between two groups. Statistical analyses were performed using Statistical Package for the Social Sciences for Windows, version 19.0 software (SPSS, Chicago, IL, USA). Differences were considered significant at $P<0.05$.

\section{Results}

\section{Patients}

Between July 2009 and December 2013, a total of 65 patients with advanced BTC were included in the current study. Baseline patient characteristics are reported in Table 1. The study population included 38 men and 27 women (ratio 1.41 ), with a mean age of 57.9 (range 38-78) years. Thirtytwo patients $(49.2 \%)$ had intrahepatic cholangiocarcinoma, including two patients (3.1\%) who presented with recurrent intrahepatic cholangiocarcinoma after resection of the original tumor but met the criteria for our study. Twenty-one patients $(32.3 \%)$ had extrahepatic cholangiocarcinoma involving the hilum (12 patients, $18.5 \%$ ) or the main bile duct (nine patients, $13.8 \%$ ). In addition, 12 patients (18.5\%) had gallbladder cancer. Spread to the regional nodes was observed in 32 patients $(49.2 \%)$. Liver metastases occurred in 21 patients $(32.3 \%)$. Biliary drainage prior to TACE was performed 
Table I Patient and tumor characteristics

\begin{tabular}{ll}
\hline Characteristic & Patients, $\mathbf{n}(\%)$ \\
\hline Age (years) & \\
Median & 58 \\
Range & $38-78$ \\
Sex & \\
$\quad$ Male & $38(58.5)$ \\
$\quad$ Female & $27(41.5)$ \\
ECOG performance status & \\
0 & $28(43.1)$ \\
I & $33(50.8)$ \\
2 & $4(6.2)$ \\
Tumor location & \\
Intrahepatic cholangiocarcinoma & $32(49.2)$ \\
Extrahepatic cholangiocarcinoma & $21(32.3)$ \\
Hilum & $12(18.5)$ \\
Main bile duct & $9(13.8)$ \\
Gallbladder cancer & $12(18.5)$ \\
Child-Pugh classification & \\
A & $50(76.9)$ \\
B & $15(23.1)$ \\
C & 0 \\
Lymph node metastases & $28(43.1)$ \\
Liver metastases & $21(32.3)$ \\
Recurrence after resection & $2(3.1)$ \\
Cancer antigen I9-9 level, U/mL, median (range) & 580.5 \\
Biliary drainage prior to TACE & $(<37-5,836.8)$ \\
\hline
\end{tabular}

Abbreviations: ECOG, Eastern Cooperative Oncology Group; TACE, transarterial chemoembolization.

in 18 patients $(27.7 \%)$. The main elevated tumor marker was cancer antigen 19-9, with a median level of 580.5 (range $<37-5,836.8$ ) U/mL. Carcinoembryonic antigen and $\alpha$-fetoprotein levels were mostly in the normal range. The Child-Pugh class was A in 50 patients and B in 15 patients, with no class $\mathrm{C}$ patients.

\section{Treatments, efficacy, and safety}

In total, 182 TACE cycles (mean 2.8 cycles per patient) were performed. All patients were evaluated for tumor response to TACE. Of the 65 study patients, 19 (29.2\%) achieved partial remission (Figure 1), 36 (55.4\%) showed stable disease, and ten (15.4\%) showed progressive disease. Thus, the overall response rate was $29.2 \%$. The condition of the patients who underwent biliary drainage prior to TACE improved significantly after the treatments. At the end of the study, only five patients were still alive. The median overall survival was 12.0 months $(95 \%$ confidence interval [CI] 8.5-15.5; Figure 2). The survival rate was significantly higher in patients who showed a tumor response to TACE than in those who showed no response (median survival 20.1 versus 9.2 months; $P<0.001$ ).
The most common adverse events involved peripheral nerve, hematological, and gastrointestinal toxicity (Table 2). Peripheral nerve and hematological toxicities were mild. Grade I-II elevations were observed in serum transaminase levels in 18 patients $(27.7 \%)$ and in total bilirubin levels in five patients $(7.7 \%)$. Only $9.2 \%$ of patients had grade III-IV adverse events (grade III-IV thrombocytopenia, three patients; grade III-IV vomiting, three patients).

\section{Discussion}

BTCs are relatively rare tumors, and are usually unresectable because of the advanced stage of disease at diagnosis. They are associated with poor clinical outcome and poor prognosis. BTCs are generally believed to be chemoinsensitive, and only a few chemotherapy regimens for BTCs are used in clinical practice. Gemcitabine is the main cytostatic agent used to treat BTC. ${ }^{19}$ To improve its therapeutic efficacy, many randomized trials have investigated gemcitabine-based combination regimens by adding a second drug, such as 5-fluorouracil, irinotecan, oxaliplatin, capecitabine, or cisplatin.

In a multicenter, randomized controlled Phase III study conducted by Valle et $\mathrm{al}^{8} 410$ patients with locally advanced or metastatic cholangiocarcinoma, gallbladder cancer, or ampullary cancer were divided into a gemcitabine-cisplatin combination arm and a gemcitabine monotherapy arm. The combination therapy improved overall survival and progression-free survival by $30 \%$ more than did gemcitabine alone, with a similar incidence of adverse events. Further, both progression-free survival and tumor control rate were significantly improved by the combination therapy. ${ }^{8}$

A retrospective study conducted by Woo et $\mathrm{al}^{20}$ which enrolled 344 patients with intrahepatic cholangiocarcinoma, gallbladder cancer, or extrahepatic cholangiocarcinoma, demonstrated that gemcitabine plus cisplatin was superior to capecitabine plus cisplatin in prolonging median overall survival. Overall survival was 8.4 months (95\% CI 6.2-10.7) in the gemcitabine plus cisplatin group and 7.6 months (95\% CI 6.8-8.7) in the capecitabine plus cisplatin group $(P=0.024)$. Based on these results, the combination of gemcitabine plus cisplatin (or oxaliplatin as a potentially better tolerated agent) is considered to be the standard of care as the first-line regimen for BTC.

TACE is considered to be a promising and minimally invasive therapeutic modality that may prolong the survival of patients with unresectable carcinomas, such as hepatocellular carcinoma, liver metastasis, or other neoplasms of the liver. One major advantage of organ-targeted delivery of agents via intra-arterial administration compared with 

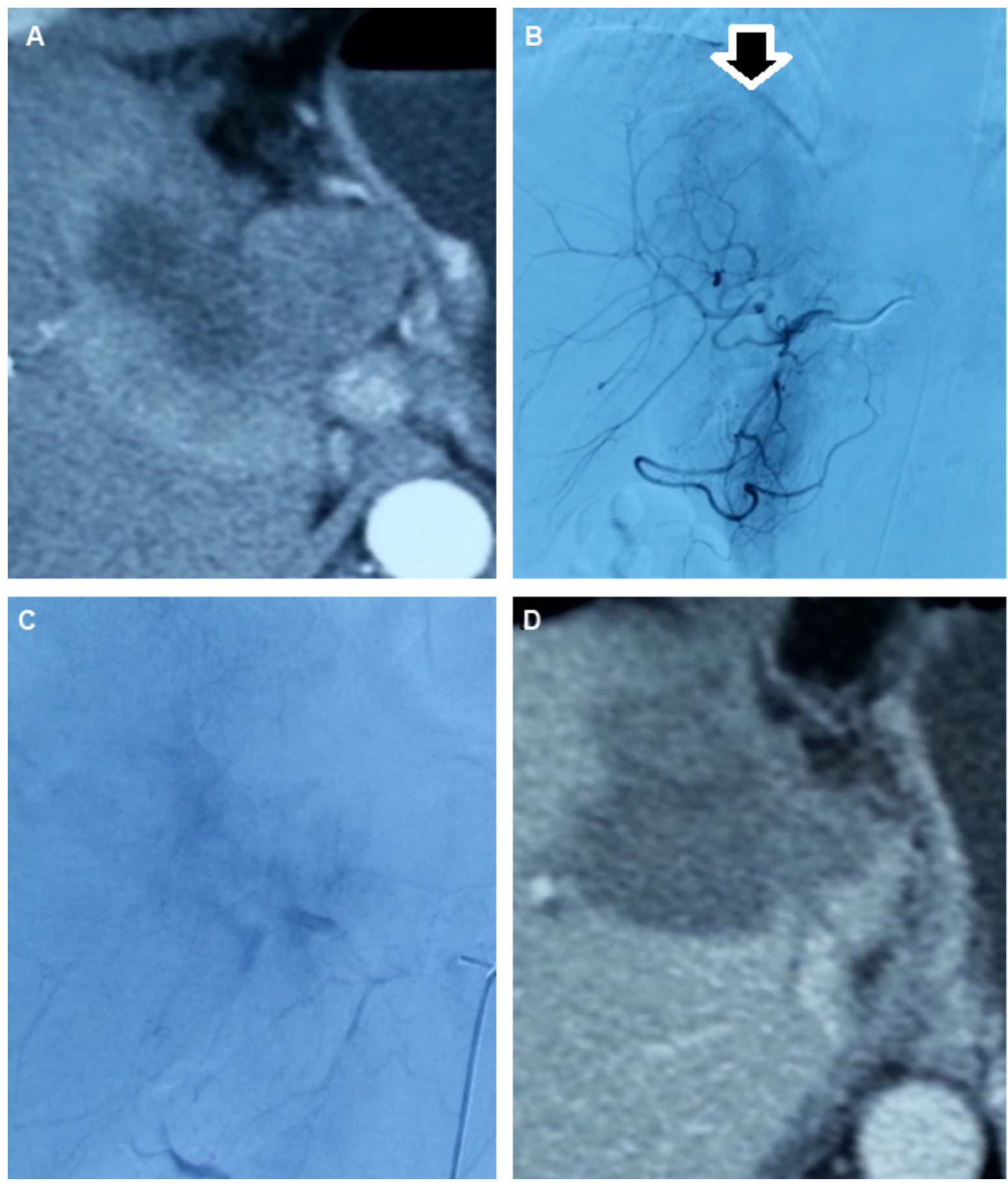

Figure I (A) Computed tomography scan showing a large cholangiocarcinoma involving the right anterior lobe and caudate lobe. (B) Digital subtraction angiography before TACE clearly demonstrates the hypervascularity of the lesion (black arrow). (C) Digital subtraction angiography after TACE shows that the feeding arteries have been mostly embolized, and the lesion is indistinct. (D) Computed tomography scan taken one month after TACE shows extensive tumor necrosis in the right anterior lobe and caudate lobe, especially in the marginal area where viable tumor existed before treatment.

Abbreviation: TACE, transarterial chemoembolization.

intravenous administration is the reduction of toxic effects on normal tissues due to reduced bioavailability of drugs in the systemic circulation. ${ }^{21}$

In this study, we have reported our experience in treating patients affected by advanced BTC with TACE involving the gemcitabine-oxaliplatin combination. The median overall survival in our study was 12.0 months (95\% CI 8.5-15.5). This result is comparable with that reported by Gusani et al. ${ }^{22}$ In their study, median survival was 13.8 months in patients with unresectable cholangiocarcinoma treated with TACE 


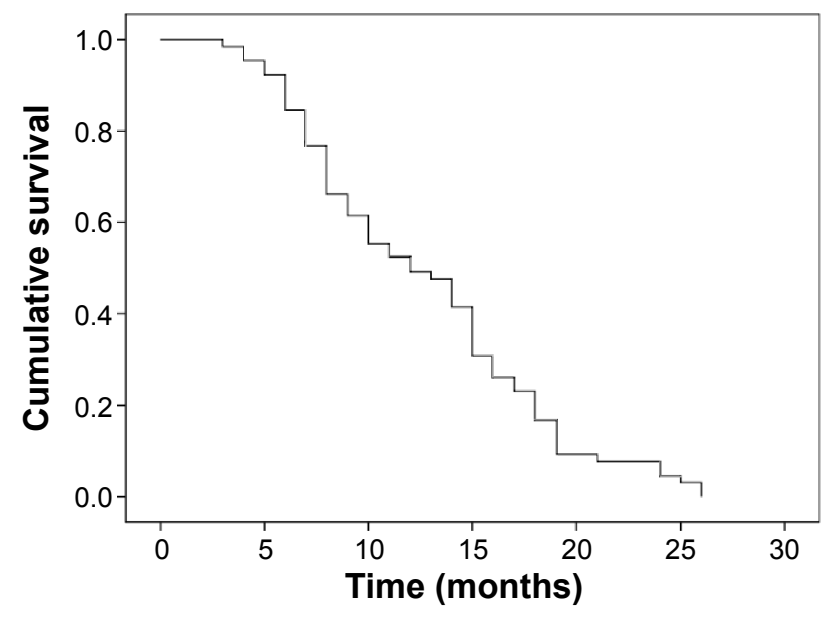

Figure 2 Survival curve for 65 patients with biliary tract cancer after transarterial chemoembolization with gemcitabine and oxaliplatin.

including the gemcitabine-cisplatin combination, which was significantly longer than the median survival after TACE with gemcitabine alone (6.3 months). Studies of intravenous chemotherapy with gemcitabine and oxaliplatin for advanced BTC have reported median survival times ranging from 8.3 to 11.0 months and response rates ranging from $14.9 \%$ to $30.8 \%{ }^{23}$ Using TACE, we achieved efficacy similar to that achieved by systemic chemotherapy. Although the difference in survival may be partly attributable to the selection bias inherent in a retrospective study, our results do indicate a benefit from the gemcitabine-oxaliplatin combination administered via TACE.

Notably, the median survival in our study was not significantly different between patients with liver disease only and those with lymph node metastases. This provides evidence that in patients with predominantly liver disease, eventual survival is determined by the liver tumors. ${ }^{22}$ Thus, regional treatment should be undertaken in these patients.

There have been studies of TACE using different combination regimens in patients with advanced BTC. Lipiodol and mitomycin $\mathrm{C}$ have been used by Scheuermann et al. ${ }^{12}$ The median survival in their study was 11 months, and the

Table 2 Adverse effects of transarterial chemoembolization with gemcitabine and oxaliplatin

\begin{tabular}{lll}
\hline Adverse effect & Grade, $\mathbf{n}(\%)$ \\
\cline { 2 - 3 } & I-II & III-IV \\
\hline Vomiting & $33(50.8)$ & $3(4.6)$ \\
Thrombocytopenia & $21(32.3)$ & $3(4.6)$ \\
Neutropenia & $20(30.8)$ & 0 \\
Peripheral nerve toxicity & $6(9.2)$ & 0 \\
Elevated serum transaminase & $I 8(27.7)$ & 0 \\
Elevated serum total bilirubin & $5(7.7)$ & 0 \\
\hline
\end{tabular}

one-year, 3-year, and 5-year survival rates were 42\%, 15\%, and $9 \%$, respectively. The median survival of 12.0 months in our study is slightly longer than that reported in their study.

In our study, only $11 \%$ of patients had grade III-IV adverse events, and no treatment-related death was recorded. The gemcitabine-oxaliplatin combination administered via TACE proved to be well tolerated, with minimal major adverse effects.

A potential limitation of our study is the heterogeneity of the BTCs included. The clinical courses of intrahepatic cholangiocarcinoma, extrahepatic cholangiocarcinoma, and gallbladder tumors are different, and the range of clinical symptoms varies considerably. Gallbladder cancers are generally less chemosensitive than extrahepatic cholangiocarcinomas. Other limitations include the retrospective non-randomized design and limited number of patients.

\section{Conclusion}

In conclusion, TACE with the gemcitabine-oxaliplatin combination is feasible and exhibits reasonable efficacy in patients with advanced BTC. Further prospective, controlled, and randomized studies with a large sample size are required to fully evaluate TACE with the gemcitabine-oxaliplatin combination for advanced BTC.

\section{Disclosure}

The authors report no conflicts of interest in this work.

\section{References}

1. Shaib Y, El-Serag H. The epidemiology of cholangiocarcinoma. Semin Liver Dis. 2004;24:115-125.

2. Tamandl D, Herberger B, Gruenberger B, Puhalla H, Klinger M, Gruenberger $\mathrm{T}$. Influence of hepatic resection margin on recurrence and survival in intrahepatic cholangiocarcinoma. Ann Surg Oncol. 2008;15:2787-2794.

3. Gruenberger B, Schueller J, Heubrandtner U, et al. Cetuximab, gemcitabine, and oxaliplatin in patients with unresectable advanced or metastatic biliary tract cancer: a phase 2 study. Lancet Oncol. 2010;11:1142-1148.

4. Pracht M, Le Roux G, Sulpice L, et al. Chemotherapy for inoperable advanced or metastatic cholangiocarcinoma: retrospective analysis of 78 cases in a single center over four years. Chemotherapy. 2012;58:134-141

5. Eckel F, Schmid RM. Chemotherapy and targeted therapy in advanced biliary tract carcinoma: a pooled analysis of clinical trials. Chemotherapy. 2014;1:13-23.

6. Hasegawa S, Ikai I, Fujii H, Hatano E, Shimahara Y. Surgical resection of hilar cholangiocarcinoma: analysis of survival and postoperative complications. World J Surg. 2007;6:1258-1265.

7. Ramírez-Merino N, Aix SP, Cortés-Funes H. Chemotherapy for cholangiocarcinoma: an update. World J Gastrointest Oncol. 2013;7:171-176.

8. Valle J, Pereira SP, Roughton M, et al. Cisplatin plus gemcitabine versus gemcitabine for biliary tract cancer. $N$ Engl J Med. 2010; 14:1273-1281. 
9. Weigt J, Malfertheiner P. Cisplatin plus gemcitabine versus gemcitabine for biliary tract cancer. Expert Rev Gastroenterol Hepatol. 2010;4:395-397.

10. Jang JS, Lim HY, Hwang IG, et al. Gemcitabine and oxaliplatin in patients with unresectable biliary cancer including gall bladder cancer: a Korean cancer study group phase II trial. Cancer Chemother Pharmacol. 2010;4:641-647.

11. Ghiringhelli F, Lorgis V, Vincent J, Ladoire S, Guiu B. Hepatic arterial infusion of gemcitabine plus oxaliplatin as second-line treatment for locally advanced intrahepatic cholangiocarcinoma: preliminary experience. Chemotherapy. 2013;59:354-360.

12. Scheuermann U, Kaths JM, Heise M, et al. Comparison of resection and transarterial chemoembolisation in the treatment of advanced intrahepatic cholangiocarcinoma - a single-center experience. Eur J Surg Oncol. 2013;6:593-600.

13. Poggi G, Amatu A, Montagna B, et al. OEM-TACE: a new therapeutic approach in unresectable intrahepatic cholangiocarcinoma. Cardiovasc Intervent Radiol. 2009;32:1187-1192.

14. Wu ZF, Zhang HB, Yang N, Zhao WC, Fu Y, Yang GS. Postoperative adjuvant transcatheter arterial chemoembolisation improves survival of intrahepatic cholangiocarcinoma patients with poor prognostic factors: results of a large monocentric series. Eur J Surg Oncol. 2012;7:602-610.

15. Park SY, Kim JH, Yoon HJ, Lee IS, Yoon HK, Kim KP. Transarterial chemoembolization versus supportive therapy in the palliative treatment of unresectable intrahepatic cholangiocarcinoma. Clin Radiol. 2011;4:322-328.

16. Shen WF, Zhong W, Liu Q, Sui CJ, Huang YQ, Yang JM. Adjuvant transcatheter arterial chemoembolization for intrahepatic cholangiocarcinoma after curative surgery: retrospective control study. World J Surg. 2011;9:2083-2091.
17. Aliberti C, Benea G, Tilli M, Fiorentini G. Chemoembolization (TACE) of unresectable intrahepatic cholangiocarcinoma with slow-release doxorubicin-eluting beads: preliminary results. Cardiovasc Intervent Radiol. 2008;5:883-888.

18. Herber S, Otto G, Schneider J, et al. Transarterial chemoembolization (TACE) for inoperable intrahepatic cholangiocarcinoma. Cardiovasc Intervent Radiol. 2007;6:1156-1165.

19. Okusaka T, Ishii H, Funakoshi A, et al. Phase II study of single-agent gemcitabine in patients with advanced biliary tract cancer. Cancer Chemother Pharmacol. 2006;5:647-653.

20. Woo SM, Lee WJ, Kim JH, et al. Gemcitabine plus cisplatin versus capecitabine plus cisplatin as first-line chemotherapy for advanced biliary tract cancer: a retrospective cohort study. Chemotherapy. 2013;3:232-238.

21. Shamseddine AI, Khalifeh MJ, Mourad FH, et al. Comparative pharmacokinetics and metabolic pathway of gemcitabine during intravenous and intra-arterial delivery in unresectable pancreatic cancer patients. Clin Pharmacokinet. 2005;9:957-967.

22. Gusani NJ, Balaa FK, Steel JL, et al. Treatment of unresectable cholangiocarcinoma with gemcitabine-based transcatheter arterial chemoembolization (TACE): a single-institution experience. J Gastrointest Surg. 2008;1:129-137.

23. Romiti A, D'Antonio C, Zullo A, et al. Chemotherapy for the biliary tract cancers: moving toward improved survival time. J Gastrointest Cancer. 2012;3:396-404.
OncoTargets and Therapy

\section{Publish your work in this journal}

OncoTargets and Therapy is an international, peer-reviewed, open access journal focusing on the pathological basis of all cancers, potential targets for therapy and treatment protocols employed to improve the management of cancer patients. The journal also focuses on the impact of management programs and new therapeutic agents and protocols on

\section{Dovepress}

patient perspectives such as quality of life, adherence and satisfaction The manuscript management system is completely online and includes a very quick and fair peer-review system, which is all easy to use. Visit http://www.dovepress.com/testimonials.php to read real quotes from published authors. 